\title{
K wie Konsequenzen eines weiteren Standortes
}

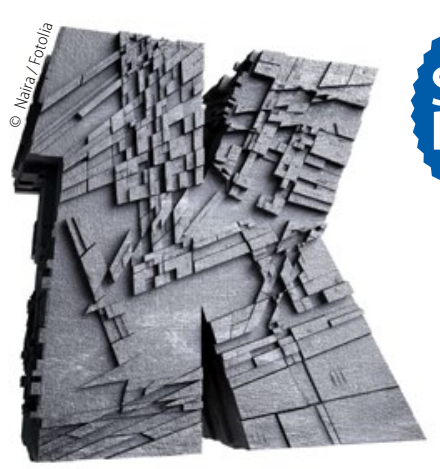

\begin{abstract}
Der Trend zur Expansion ist ungebrochen. Viele Ärzte planen die Übernahme einer Zweitpraxis, was meist durch eine überörtliche Berufsausübungsgemeinschaft (ÜBAG) geschieht. Gerade bei der Praxisbuchhaltung führt dies zu einer Vielzahl von Fragen.
\end{abstract}

Eine Erfassung der Einnahmen pro Standort ist in der Finanzbuchhaltung nicht ohne Weiteres möglich, da die Praxis - auch wenn sie mehrere Standorte hat - nur eine gemeinsame Abrechnung für die Kassenzahnärztliche Vereinigung (KZV) vornimmt. Das heißt, die Zahlungen der KZV und eigentlich auch der Patienten erfolgen für die Gesamtpraxis und können deshalb in der Buchhaltung nicht korrekt pro Standort angezeigt werden. Eine differenzierte Betrachtung der erbrachten Leistungen pro Standort kann lediglich anhand der Leistungsstatistik der Praxissoftware erfolgen. In dieser müssen die Leistungen der Zahnärzte und Prophylaxemitarbeiter standortbezogen erfasst werden. Anhand dieser Aufteilung können dann die geflossenen Einnahmen der Buchhaltung im Rahmen einer Profitcenterrechnung prozentual verteilt werden.

\section{Zusätzliche Konten für mehr Transparenz}

Damit in der Buchhaltung die zusätzlichen Kosten aus der Erweiterung transparent abgebildet werden können, sollten im Kontenplan einige zusätzliche Konten angelegt werden. Wichtig ist, dies nur für die Kosten zu tun, bei denen auch klar differenziert werden kann, an welchem Standort sie entstanden sind. So können zum Beispiel die Mietkosten für die beiden Praxen in der Regel als Einzelkosten genau dem jeweiligen Standort zugeordnet und dafür in der Buchhaltung separate Mietkostenkonten angelegt werden. Es empfiehlt sich bei der Differenzierung der Kostenkonten für jeden Standort immer die gleiche letzte Kontenstelle zu verwenden. Man kann entweder im SKR03 das Konto 4210 Miete und Nebenkosten in zwei Unterkonten auflösen, etwa „4211 Miete und Nebenkosten - Standort A“, „4212 Miete und Nebenkosten - Standort B“. Oder man behält das bisherige Konto „4210 Miete und Nebenkosten“ für den Hauptstandort bei und legt für den neuen Standort das zusätzliche Konto „4211 Miete und $\mathrm{Ne}$ benkosten - Standort B“ an. Weitere Kostenkonten, bei denen sich eine klare Trennung nach Standort empfiehlt, sind beispielsweise Instandhaltung der Praxisräume, Energiekosten, Sonstige Raumkosten, Dekoration, Instandhaltung Einrichtung, Gerätemiete und Leasing, Telefongebühren und ähnliche. Für jede Praxis muss die Festlegung mit Blick auf die zugrunde liegende Organisationsstruktur individuell erfolgen.

\section{Verteilungsrechnung für weitere Kosten}

Kosten, die nicht einem einzelnen Standort zugeordnet werden können, werden als Gemeinkosten bezeichnet und müssen in Form einer Verteilungsrechnung auf die Standorte umgelegt werden. Hierfür empfiehlt sich die Anlage einer Profitcenterrechnung, bei der Gemeinkosten nach festgelegten Strukturen oder aufgrund der Datenbasis dynamisch zugeordnet werden. In aller Regel fallen bei Zahnarztpraxen hierunter beispielsweise die Personalkosten, denn die monatliche Gehaltsabrechnung wird ebenfalls für die gesamte Praxis erstellt und nicht pro Standort. Das Personal kommt meist an mehreren Standorten zum Einsatz und könnte, selbst wenn die Gehaltsabrechnungen getrennt würden, nicht eindeutig einem Standort zugeordnet werden.

Nur eine differenzierte Buchhaltung ermöglicht eine dauerhafte Erfolgskontrolle, bei der die Entwicklung je Standort betrachtet werden kann.

Mehr Infos unter www.fibu-doc.de

Das in den Artikeln dargestellte Buchhaltungskonzept basiert auf der zahnarztspezifischen Buchhaltungssoftware fibu-doc und wird vom FVDZ unterstützt.
Seminare
Personalkosten in der Zahnarztpraxis
12.10.2016 Düsseldorf
Das kosten unsere Mitarbeiter wirklich
Praxisbuchhaltung - leicht und
verständlich (Theorie)
14.10.2016 Münster
Der Einstieg in die Buchhaltung mit
fibu-doc (Praxis)
Praxisgründung - betriebswirtschaftliche $\quad$ 19.10.2016 Kassel Grundsätze
Der Blick in die Praxiszahlen 26.10.2016 Düsseldorf
Personalkosten in der Zahnarztpraxis
Das kosten unsere Mitarbeiter wirklich
11.11.2016 Berlin

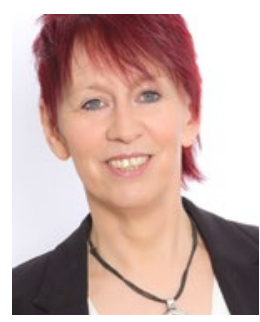

Barbara Mertens

FIBU-doc Praxismanagement GmbH 